\title{
A note on the cyclic evolution of the pulsar magnetosphere (Research Note)
}

\author{
I. Contopoulos
}

Research Center for Astronomy, Academy of Athens, 11527 Athens, Greece

e-mail: icontop@academyofathens.gr

Received 18 June 2007 / Accepted 10 September 2007

\section{ABSTRACT}

\begin{abstract}
Positive and negative pulsar breaking indices suggest that some fraction of the pulsar spindown torque undergoes a cyclic evolution. The observed strong correlation of "anomalous" breaking indices with pulsar age implies that the characteristic periodicity timescale is in the range 100 to 10000 years depending on the fraction of the spindown torque that undergoes cyclic evolution, 1 to $100 \%$ respectively. We argue that the longest variability timescale is consistent with a neutron star magnetic cycle similar to the solar cycle.
\end{abstract}

Key words. stars: pulsars: general - stars: magnetic fields

\section{Observational evidence}

A pulsar spins down due to the torque on the neutron star crust generated by the electric current flowing in its magnetosphere. In the simplified picture of a steady-state axisymmetric force-free ideal MHD magnetosphere, Contopoulos et al. (1999, hereafter CKF) first showed that the distribution of the magnetospheric electric current $I$ can be determined as an eigenvalue of the problem, if one makes the natural assumption that the magnetosphere is smooth and continuous on the light cylinder (defined as the distance $r_{\mathrm{L}} \equiv c / \Omega$ from the rotation axis, where $\Omega$ is the pulsar angular velocity). The unique electric current distribution thus obtained yields a unique pulsar spindown torque, and thus a unique pulsar spindown rate $\dot{\Omega}$. This is of the same order as the value obtained for simple electromagnetic vacuum dipole radiation, namely

$\dot{\Omega}=-f \frac{B_{*}^{2} r_{*}^{4}}{c^{3} M_{*}} \Omega^{3}$.

Here, $B_{*}, r_{*}$, and $M_{*}$ are the neutron star polar magnetic field, radius and mass respectively; and $f$ is a numerical factor of order unity. It turns out that this result is also valid in the general non-axisymmetric case (Bogovalov 1999; Spitkovsky 2006; Contopoulos 2007a), and therefore, one may use Eq. (1) to obtain an estimate of the polar value of the neutron star magnetic field from measurements of $\Omega$ and $\dot{\Omega}$.

Strictly speaking, however, the results of CKF and subsequent related work are only valid in steady-state. Thus, as the neutron star spins down and the light cylinder moves to larger and larger distances, one needs to take into account the evolution of the pulsar magnetosphere. The first thing one may assume is that the magnetosphere evolves through a sequence of steadystate equilibria of the CKF type, i.e. that it manages to readjust itself so that at all times, the region of closed lines extends all the way to the light cylinder, and the last open magnetic field line extends to infinite distances without reconnecting accross the equator. In addition, one may assume that $B_{*}$ does not evolve

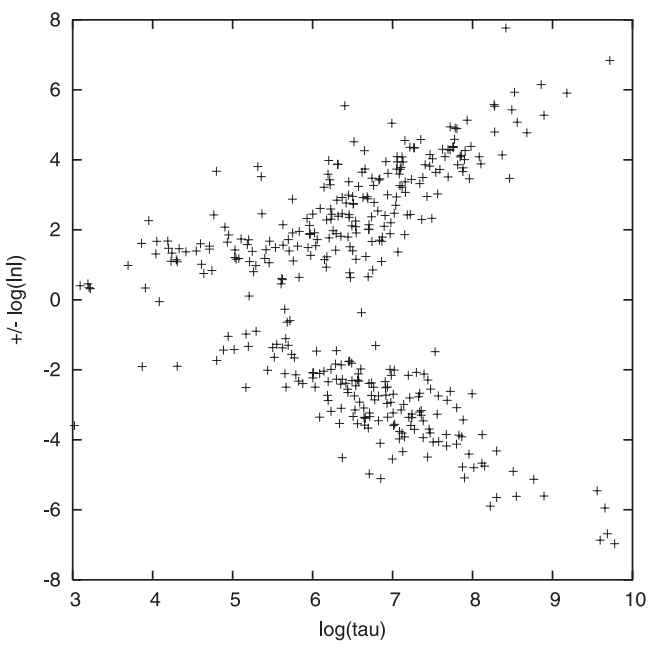

Fig. 1. Braking index as a function of characteristic spindown time. We plot here $\pm \log |n|$ ( \pm according to whether $n>0$ or $n<0)$ vs. $\log \tau$, where $\tau \equiv-\Omega / 2 \dot{\Omega}$ in years. Note that $|n|>1$ everywhere. At large $\tau>10^{5}$, the diagram may be fit by the simple linear relation $\pm \log |n| \sim$ $\pm(\log \tau-3)$.

with pulsar age. Unfortunately, the situation is more complicated than that, since Eq. (1) yields a braking index value

$n \equiv \frac{\ddot{\Omega} \Omega}{\dot{\Omega}^{2}}=3$,

and most known measurements of $n$ differ from that value. In fact, there exist today $\sim 400$ pulsars in the ATNF catalogue (Manchester et al. 2005) with measured values of $n$ in the range from $-10^{6}$ to $+10^{6}$. Although all but six of these values are characterized in the literature as "anomalous", one thing is certain: Eq. (2) cannot be right in general.

In Fig. 1 we plot $\pm \log |n|$ (with \pm according to whether $n>$ 0 or $n<0$ respectively) as a function of the characteristic spindown time $\tau \equiv-\Omega /(2 \dot{\Omega})$ in years. One may argue (Alice Harding, personal communication) that in young pulsars 


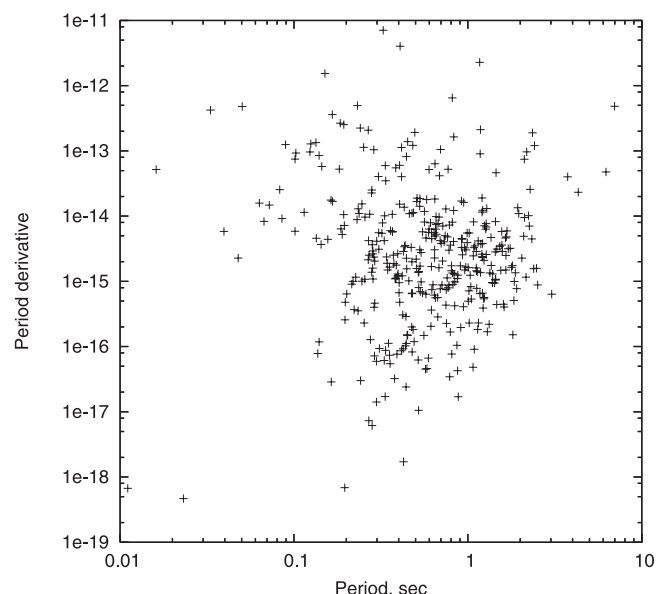

Fig. 2. $P-\dot{P}$ diagram for the $\sim 400$ pulsars with measured braking index values shown in Figure 1 . Here, $P, \dot{P}$ are the pulsar period and period derivative respectively. The distribution is that of a standard sparse $P-\dot{P}$ diagram without any obvious observational bias.

( $\tau<10^{5}$ ), braking index measurements may be "corrupted" by neutron star glitches. On the other hand, in older pulsars $(\tau>$ $10^{5}$ ) where glitches are not as important, one finds the correlation

$\pm \log |n| \sim \pm(\log \tau-3)$.

Moreover, in a $P-\dot{P}$ diagram $(P \equiv 2 \pi / \Omega$ is the pulsar period $)$ containing all the cases with observed values of $n$, there is no obvious correlation between $P$ and $\dot{P}$ (Fig. 2). In other words, the pulsars with measured values of $n$ have been taken randomly from the total number of known pulsars without any obvious observational bias, and therefore, they represent pulsars at various stages of their evolution. Beskin et al. (2006) argued that the \pm symmetry of Fig. 1 implies that some fraction of the pulsar angular velocity undergoes a cyclic evolution. We suggest that it is more constructive to consider a cyclic component in the evolution of the pulsar spindown torque (which is, after all, the source of the angular velocity evolution). Interestingly enough, as we will see next, the data also yields some information on the periodicity timescale.

There are several ways to reconcile Eqs. (1) and (3): (A) assume that the approximation of a sequence of CKF-type steadystate magnetospheric equilibria holds, and that the neutron star magnetic field undergoes a cyclic evolution; (B) relax the assumptions of the CKF analysis and assume a variable magnetospheric structure that would yield a cyclic evolution of the factor $f$; (C) relax the assumption of constant neutron star moment of inertia. Only (A) and (B) refer to the spindown torque itself. In any case,

$\dot{\Omega}=-f_{\mathrm{o}} \frac{B_{* 0}^{2} r_{*}^{4}}{c^{3} M_{*}} \Omega^{3} F(t)$,

with $f_{\mathrm{o}}, B_{* \mathrm{o}}$ characteristic values of the spindown parameter $f$ and the polar magnetic field $B_{*}$ respectively, and

$F(t)=1-\frac{\alpha}{2}+\frac{\alpha}{2} \cos \left(2 \pi \frac{t}{\tau_{\text {cycle }}}+\phi\right)$

characterizing the cyclic variation of either $f$ or $B_{*}$. Here, $\tau_{\text {cycle }}$ is the characteristic period of the cyclic spindown evolution in years; $\alpha$ is the fraction of the spindown torque that varies periodically $(0 \leq \alpha \leq 100 \%)$; and $\phi$ is a random initial (at pulsar

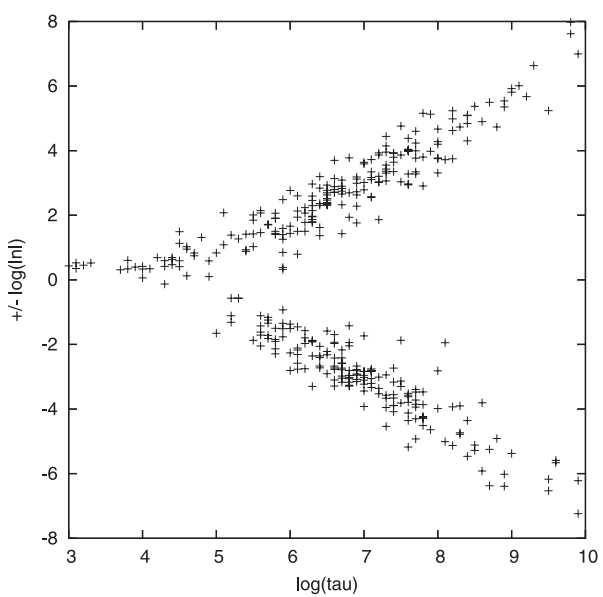

Fig. 3. Fit of the distribution shown in Fig. 1 assuming a 100\% cyclic evolution of the pulsar spindown torque (Eqs. (4) and (5)). $\tau_{\text {cycle }} \sim$ 10000 (in years). The fit is acceptable, even for young pulsars $\left(\tau \leq 10^{5}\right)$ where some of the dispersion in the measurements of $n$ is due to neutron star glitches.

birth) phase angle. Note that, in order for the star to continuously spin down we must have $F \geq 0$ at all times. Therefore,

$n=3+\frac{\Omega / \dot{\Omega}}{F / \dot{F}} \sim 3+2 \pi \alpha \frac{\tau}{\tau_{\text {cycle }}}$.

For old pulsars with $\tau \gtrsim \tau_{\text {cycle }}$, Eq. (6) can equivalently be written as

$\pm \log |n| \sim \pm\left(\log \tau-\log \tau_{\text {cycle }}+1+\log \alpha\right)$.

Comparing Eqs. (3) and (7), one obtains the following approximate relation between the characteristic period and the fraction of the pulsar spindown which varies in a cyclic way,

$\log \tau_{\text {cycle }} \sim 4+\log \alpha$.

We plot in Fig. 3 what Eq. (6) yields for the $\sim 400$ pulsars with measured values of $n$, assuming $\alpha=100 \%$. Note that the fit is independent of $f_{\mathrm{o}}$ and $B_{*_{\mathrm{o}}}$. As Beskin et al. (2006) suggested, the minimum characteristic period cannot be smaller than the pulsar observation period of 40 years, and this together with Eq. (8) yields a range $100 \lesssim \tau_{\text {cycle }} \lesssim 10000$ for $1 \% \leq \alpha \leq 100 \%$ respectively.

\section{Cyclic magnetospheric evolution}

Several physical models that address the issue of cyclic variation of the pulsar spindown have been proposed in the literature, ranging from neutron star interior "wobbling" on a timescale of a few years (e.g. Kundt 1988), to magnetospheric variability (e.g. Contopoulos 2005). In the present work, we would like to focus on our simplest (one-parameter) fit of the anomalous braking index data, namely the one with $\tau_{\text {cycle }} \sim 10000$ and $\alpha \approx 100 \%$.

$F(t)$ becoming zero periodically is not compatible with a cyclic evolution of the neutron star moment of inertia (case $\mathrm{C}$ above). On the other hand such a scenario is compatible with a cyclic evolution of the neutron star magnetic field similar to the solar cycle (case A above). Interestingly enough, the ten thousand year timescale that we obtain is comparable to the neutron star cooling timescale (e.g. Blandford et al. 1983). It is conceivable that some sort of dynamo mechanism in the neutron star interior, may support a cyclic evolution with

$B_{*}=B_{*_{\mathrm{o}}} \cos \left(2 \pi \frac{t}{20000 \text { years }}+\phi\right)$ 

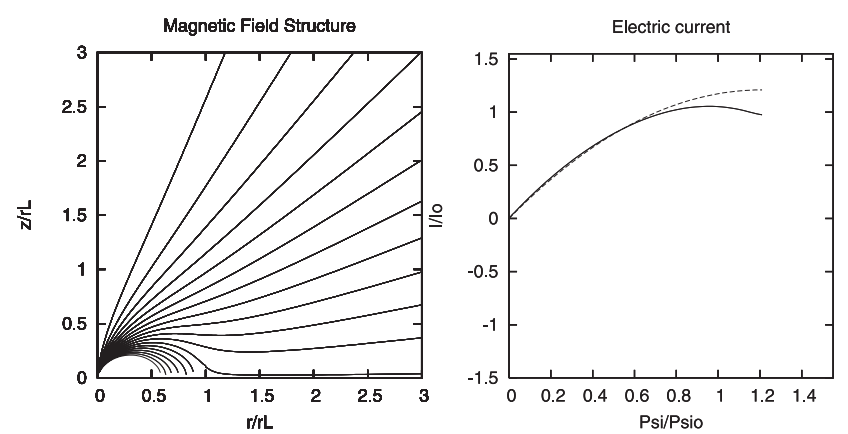

Fig. 4. On the left, we plot the magnetic field structure in the case of no magnetospheric reconnection (CKF). Distances are normalized to the light cylinder distance $r_{\mathrm{L}}$. On the right, we plot the corresponding electric current distribution $I=I(\Psi)$ along the field lines that cross the light cylinder. The magnetic flux is normalized to the canonical value $\Psi_{o} \equiv \pi B_{*} r^{3} / r_{\mathrm{L}}$. The electric current is normalized to the canonical value $I_{o} \equiv \Omega \Psi_{o} /(4 \pi)$. For comparison, we plot also (dashed line) the electric current distribution of a relativistic magnetic split monopole with the same amount of magnetic flux crossing the light cylinder (Michel 1991).
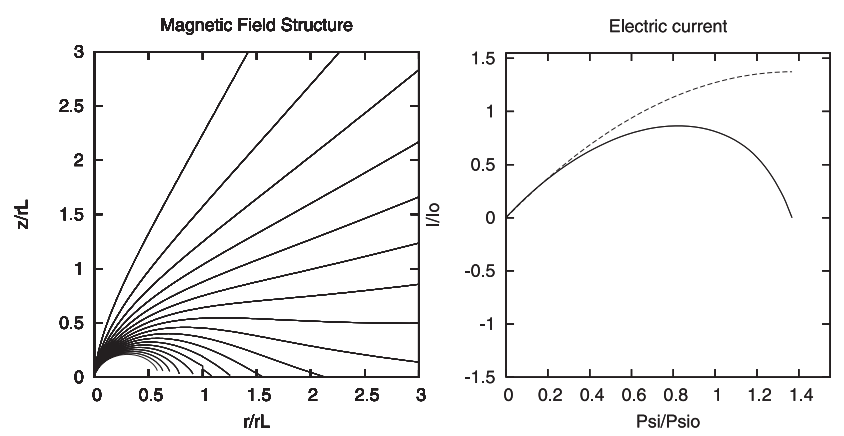

Fig. 5. Same as Fig. 4 with some amount of equatorial magnetospheric reconnection that corresponds to $\epsilon=0.4$ (Eq. (10)).

(Eqs. (4), (5)). Note that this scenario does not require magnetic field decay (at least over timescales shorter than about $10^{11}$ years), in agreement with the analysis of the $P-\dot{P}$ diagram presented in Contopoulos \& Spitkovsky (2006).

We also tried to seek variants of the CKF solution (case B above) that would yield values of $f$ very different from unity. In fact, what we need is a physical mechanism that will periodically turn off the neutron star magnetospheric spindown. In a series of papers (Contopoulos 2007b,c), we relaxed the assumption of ideal MHD in the equatorial region of the pulsar magnetosphere beyond the light cylinder. This is the region where the magnetospheric return current flows, and several authors before us suggested that this may be the region of electromagnetic energy dissipation that would result in particle acceleration (e.g. Coroniti 1990; Michel 1994; Lyubarsky \& Kirk 2001; Kirk \& Skjæraasen 2003; Romanova, Chulsky \& Lovelace 2005). As we argued in Contopoulos (2007c), one cannot study equatorial reconnection without taking into account the global topology of the poloidal magnetic field. The details of equatorial reconnection remain (yet) unknown. However, it is easy to realize that, when equatorial reconnection is present, magnetic field lines that cross the light cylinder and would have extended to infinity in $\mathrm{CKF}$, now continuously reconnect across the equator. As a result, the equatorial condition for the magnetic flux function $\Psi(r ; z)$ (defined as the magnetic flux crossing a circle of cylindrical radius $r$ at height $z$ around the axis of rotation) differs from that in CKF. In particular, $\Psi\left(r>r_{\mathrm{L}} ; z=0\right)$ is not constant but decreases with distance. We assume for simplicity that

$\Psi\left(r>r_{\mathrm{L}} ; z=0\right)=\Psi\left(r=r_{\mathrm{L}} ; z=0\right)\left(r / r_{\mathrm{L}}\right)^{-\epsilon}$,
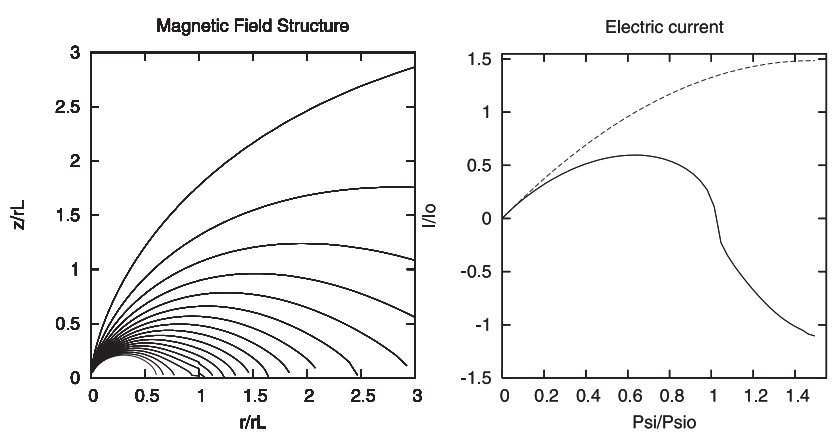

Fig. 6. Same as Fig. 4 with maximum equatorial magnetospheric reconnection that corresponds to $\epsilon=1$.

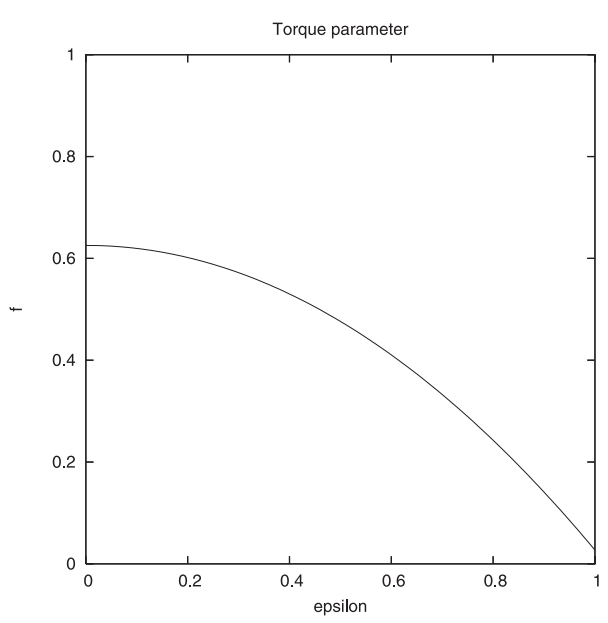

Fig. 7. The spindown torque parameter $f$ (Eq. (1)) as a function of the dissipation parameter $\epsilon$. In the absence of reconnection $(\epsilon=0), f=0.6$ (CKF).

where, $\epsilon$ is a parameter that characterizes the effect of dissipation $(\epsilon=0$ corresponds to the ideal MHD case studied in $\mathrm{CKF}$, whereas $\epsilon=1$ corresponds to a magnetosphere with maximum equatorial dissipation). Equation (10) is a new (to our knowledge) equatorial boundary condition beyond the light cylinder, and one may thus implement the same procedure as described in CKF to solve the pulsar equation (Scharlemann \& Wagoner 1973), and thus obtain the magnetospheric structure and electric current distribution $I(\Psi)$ for various values of $0 \leq \epsilon \leq 1$, as seen in Figs. 4-6. For each such electric current distribution the total electromagnetic spindown torque acting on the neutron-star crust is proportional to the integral $\int I(\Psi) \mathrm{d} \Psi$ (e.g. Michel 1991). Note that when $\epsilon=0.4$ (Fig. 5) the return current is distributed along the magnetic field lines that cross the light cylinder, whereas when $\epsilon=1$ (Fig. 6) $\int I(\Psi) \mathrm{d} \Psi \sim 0$, i.e. the total neutron-star spindown torque is close to zero. In Fig. 7 we plot the value of the spindown torque parameter $f$ as a function of our dissipation parameter $\epsilon$. One sees that, as we introduce more and more dissipation in the equatorial region, the magnetosphere evolves to a configuration with less and less electromagnetic torque acting on the central neutron star. Obviously, a cyclic evolution of the physical mechanism that allows or inhibits equatorial dissipation in the pulsar magnetosphere (e.g. variability in the supply of charge carriers from the neutron star surface that may be due to a periodic stellar wind) would yield a cyclic evolution of the magnetospheric torque. Unfortunately, magnetospheric solutions for $0.4 \leq \epsilon \leq 1$ ( $f \leq 0.5$, or equivalently $F \leq 80 \%$ ) contain regions with $I(\Psi)<0$, where electromagnetic energy is flowing from the magnetosphere onto the star, and therefore, such solutions are probably unphysical. 
We conclude that a cyclic component in the evolution of the magnetospheric spindown torque may account for the measured large positive and negative anomalous braking index values. If we are willing to consider a $100 \%$ cyclic evolution, this can only be due to a neutron star magnetic cycle similar to the solar cycle. In that case, the evolution timescale would be on the order of 10000 years.

Acknowledgements. We would like to thank Drs. Alice Harding and Demos Kazanas for their hospitality at the NASA Goddard Space Flight Center in January and June 2007 where some of the ideas in the present work originated. We would also like to thank Pr. Wolfgang Kundt for an honest exchange of ideas.

\section{References}

Beskin, G., Biryukov, A., \& Karpov, S. 2006 [arXiv:astro-ph/0603375] Blandford, R. D., Applegate, J. H., \& Hernquist, L. 1983, MNRAS, 204, 1025
Bogovalov, S. 1999, A\&A, 349, 1017

Contopoulos, I. 2005, A\&A, 442, 579

Contopoulos, I. 2007a, in 363rd WE-Heraeus Seminar on Neutron Stars and Pulsars, ed. W. Becker, \& H. H. Huang, MPE Rep., 291, 132

Contopoulos, I. 2007b, A\&A, 466, 301

Contopoulos, I. 2007c, A\&A, 472, 219

Contopoulos, I., \& Spitkovsky, A. 2006, ApJ, 643, 1139

Contopoulos, I., Kazanas, D., \& Fendt, C. 1999, ApJ, 511, 351 (CKF)

Coroniti, F. V. 1990, ApJ, 349, 538

Kirk, J. G., \& Skjæraasen, O. 2003, ApJ, 591, 366

Kundt, W. 1988, Com. Ap., 12, 113

Lyubarsky, Y., \& Kirk, J. G. 2001, ApJ, 547, 437

Manchester, R. N., Hobbs, G. B., Teoh, A., \& Hobbs, M. 2005, AJ, 129, 1993

Michel, F. C. 1991, Theory of Neutron Star Magnetospheres (Chicago: Univ. Chicago Press)

Michel, F. C. 1994, ApJ, 431, 397

Romanova, M. M., Chulsky, G. A., \& Lovelace, R. V. E. 2005, ApJ, 630, 1020

Scharlemann, E. T., \& Wagoner, R. V. 1973, ApJ, 182, 951

Spitkovsky, A. 2006, 648, 51 